\title{
The Comorbidities of Childhood Obesity
}

Author 1: Esther Ejiroghene Ajari

Email Address: estherejiroghene@gmail.com

Affiliations:

1. Faculty of Clinical Sciences, College of Medicine, University of Ibadan, Ibadan, Oyo State, Nigeria

2. The TriHealthon, Ibadan, Oyo State, Nigeria

Author 2: Babatunde Isaac Ademusire

Email Address: babatundeademusire@gmail.com

Affiliation:

1. Faculty of Clinical Sciences, College of Medicine, University of Ibadan, Ibadan, Oyo State, Nigeria

Author 3: Abdulhammed Babatunde

Email Address: abdulhammedbabatunde99@gmail.com

Affiliation:

1. Faculty of Clinical Sciences, College of Medicine, University of Ibadan, Ibadan, Oyo State, Nigeria

Corresponding Author: Esther Ejiroghene Ajari

Address: C1, Alexander Brown Hall, University College Hospital, Ibadan, Oyo State, Nigeria Email Address: estherejiroghene@gmail.com 


\begin{abstract}
Childhood obesity is a preventable multi-systemic disorder, defined as the body mass index of a child being at or above the 95th percentile, has become an epidemic. In 2019, about 38 million under-five children and over 300 million individuals aged 5-19 were reported to be overweight or obese. In this narrative review, we showed evidences, from existing literature, of the affectation of various body systems by childhood obesity.

Co-morbid disorders associated with childhood obesity in the cardiovascular, nervous, dermatological, respiratory, endocrine, reproductive, musculoskeletal, gastrointestinal, and renal systems, and its effects on mental health are discussed. Since obesity ultimately reduces the quality of life of children and is a leading cause of preventable deaths of children globally, effective research, policy and community-based project interventions, aimed at the prevention of obesity and the treatment of its co-morbid disorders, should be taken by all appropriate stakeholders.
\end{abstract}

Keywords: Obesity, Childhood, Comorbidity, Malnutrition

\title{
Introduction
}

Obesity and overweight are nutritional disorders caused by energy imbalance between calories consumed and calories expended. Epigenetics also influences the transmission of obesity risk from parents to offspring (1). Single-nucleotide polymorphisms at genetic loci for adipokines and their receptors are linked to obesity (1).

Unlike in adults in which a body mass index (BMI) of $>30 \mathrm{~kg} / \mathrm{m} 2$ is diagnostic of obesity (2), BMI is not used to identify obesity in children. Instead, a percentile scale based on the child's sex and age is used. In this population, BMI at the 85th to 94th percentile signifies overweight while BMI at or above the 95th percentile signifies obesity (2).

There has almost been a five-time increase in the worldwide obesity since the 1970s (2). In the last three decades, the global prevalence of childhood obesity has increased by $47.1 \%$, with 38 million under-five children and over 300 million individuals aged 5-19 being reported as overweight or 
obese in 2019 (2). About 50\% of these children reside in Asia, while over 8.5\% of them reside in Africa (2).

Obesity is a multi-system disease that kills more people than underweight. These deaths are linked to the co-morbidities associated with obesity. For every 5 -unit increase in BMI above the $25 \mathrm{~kg} / \mathrm{m}^{2}$ threshold, total mortality increases by $29 \%$, vascular mortality by $41 \%$, and diabetes-related mortality by $210 \%$ (3). Measures of central adiposity, such as increased waist circumference, predict these risks (3).

In this paper, we review evidences of the various co-morbidities associated with obesity according to the body systems.

\section{Cardiovascular system}

Childhood obesity is commonly associated with cardiovascular diseases which usually manifest after puberty and varies with BMI. Studies have shown the effect of obesity on cardiac and vascular health of youths resulting in development of accelerated atherosclerosis enhanced by dyslipidaemia which is usually evident during autopsy. Atherosclerosis, increased arterial stiffness, and vascular resistance result in increased afterload. Ventricular hypertrophy, together with other cardiovascular co-morbidities, is not only peculiar to obese youths and adult but also children and this worsens as the fat mass remains persistent into adulthood (2). These cardiovascular abnormalities increase the risk of hypertension in obese children and adolescents which may pose greater risk of early organ damage because of their exposure to high blood pressure at a very early age of life $(3,4)$. The resultant organ damage is usually fatal and difficult to manage clinically.

In a study by Sorof et al. (2020), conducted to determine the prevalence of hypertension in schoolaged children, 5 percent of the over 5000 children that participated in the study were found to have persistent high blood pressure with obesity being the strongest risk factor (5). The pathophysiological mechanisms of childhood hypertension increase cardiac output and/or total peripheral resistance. Some of these mechanisms include increased sympathetic outflow, insulin resistance, renin-angiotensin-aldosterone system, vascular damage, sodium and water retention 
(6). Furthermore, impairment of cardiac structures by obesity in children also increases the risk of cardiac autonomic modulation (CAM) which results in increased sympathetic outflow and reduced parasympathetic innervation to the heart (7). This further contributes to other cardiovascular comorbidities like coronary heart disease and hypertension in obese children.

Obese children suffer from abnormal lipid profile characterized by low HDL and high LDL in the blood which increases the risk of fat deposition in coronary arteries and carotid arteries. Atherosclerosis, dyslipidaemia, structure damage of the myocardium and vascular wall are disorders associated with obesity in children and may be complicated by diabetes mellitus or family history of hypocholesterolaemia $(4,8)$. Children with atherosclerosis may not present with any clinical symptoms until adulthood when the plaque has significantly narrowed the vessel increasing the risk of developing coronary artery diseases or even sudden cardiac death as young adults. Coronary artery ultrasound, together with CIMT measurement, a non-invasive technique for measuring the intima-media thickness of coronary vessels, serves as an effective marker for early detection of atherosclerosis in children and can be useful in identifying children with higher risk of developing coronary artery disease later in life (9).

However, some children who are obese from childhood till adulthood may never develop any cardio-metabolic co-morbidity and have higher chance of remaining healthy later in life (10). This condition is known as metabolically healthy obesity (10). Although this may be rare but it affirms that not all obese children have higher risk of cardiovascular co-morbidities than normal weight children.

\section{Nervous system}

Morphological alteration in the brain may also arise as a result of obesity in children. According to a study on the difference in morphology of brain substance among children, there was significantly increased volume of amygdala and nucleus accumbens in obese children (mean age: 13.8+/-1.9) and a reduced gray matter mass without any relationship with age or gender (11). The effect of this difference has not been elucidated and more research is required to confirm this association on a larger study size. 


\section{Mental health}

Obesity not only increases the risk of cardio-metabolic disorders but also have significant effect on the psychological and social well being of children. It can either be a primary or secondary comorbidity or even both. Psychological co-morbidities of childhood obesity include depression, anxiety, low self-esteem, compromised perceived Quality of Life (QoL), behavioural disorders (12). These may be as a result of other predisposing factors such as stigmatization, isolation, "body-shaming", teasing and other negative behaviours from peers and the society in general. Hussein et al. (2020), studied the correlation between obesity and common psychiatric disorders in 60 obese (BMI >95 percentile) and 60 normal children. There was a significant correlation between obesity, depression and low self-esteem $(\mathrm{P}<0.05)$ with girls being more vulnerable than boys (13). Obesity is a recognized cause of low self-esteem in childhood, particularly among boys and white and Hispanic girls aged 13-14 years (14). Low self-esteem is a risk factor for loneliness, nervousness, smoking and alcohol intake (14). Although anxiety and oppositional defiant disorder (ODD) were also more prevalent in obese children, the difference was not statistically significant. Bullying of obese children was a major risk factor that contributed to development of depression among them (13).

Eating disorder and Attention Deficit/Hyperactivity Disorder are other comorbidities associated with childhood obesity. Overweight girls, regardless of clinical status, experience binge eating (15), more depressive symptoms (16) and have a lower quality of life compared to their peers of normal weight (17). In a study carried out on 26 school-aged children (8-17 years) hospitalized for obesity (BMI >85\%) over 4 year period, 57.7\% suffered from Attention Deficit/Hyperactivity Disorder (ADHD), a significantly higher prevalence when compared to $10 \%$ in the same age group for the general population ( $\mathrm{p}<.0001$ ), with boys to girls ratio being 2:1 (18). Loss of control (LOC) over eating, an example of an eating disorder that is associated with obesity in children, also contributes to increased adiposity in the body. This kind of reciprocal relationship between obesity and LOC is also reported for depression and ADHD because as much as children with obesity have higher prevalence of these conditions than normal weight children, those with these conditions tend to also have higher BMI than the general population (19). This can be attributed to the fact that depression, LOC and ADHD often cause inactivity, overeating, insomnia and low self-esteem 
which are factors that reduce amount of calories used daily and ultimately result in overweight/obesity.

Obese children have a higher risk of a wide range of psychosocial and mental health disorders than normal weight children. In a study of about 43,297 children between ages 10 and 17 years randomly selected by a telephone interview of parents or guardians in $2007 \mathrm{NSCH}$, there was a significant association ( $\mathrm{p}<0.001$ ) between obesity and mental and psychosocial conditions which include internalizing problem, externalizing problem, repeat grade, school problems, conduct disorder, learning disability and developmental delay together with ADHD (P .003), and depression (p .006) while anxiety was reported to have no significant association with BMI. (20)

\section{Dermatological system}

Many skin disorders are associated co-morbidities of obesity in adulthood and some have been reported also in children. Skin tags (40\%), striae distensae (32\%), plantar hyperkeratosis (20\%), hyperhidrosis (24\%) and acanthosis (12\%) were the dermatoses (percentage of subjects) that were significantly associated with obesity ( $>95$ th percentile) in a study of 65 overweight and obese children (aged 8-15years) at the Department of Dermatology, University Federico II, Naples, Italy while none of the 30 normal weight children in the control group had these dermatoses (21). There was also no observed sex difference in skin diseases in obese children (21).

According to a retrospective cohort study of 248,775 children aged 5-17 years, skin infections caused by bacteria, fungi and candidiasis were significantly associated ( $\mathrm{p}<0.001$ ) with obesity (BMI > 95th percentile) while cutaneous viral infections showed an inverse relationship. Other risk factors for bacterial infections are female sex, diabetes and white race. Pruritus, atopic dermatitis (22), psoriasis, seborrheic dermatitis, hidradenitis, hyperhidrosis and mechanical cutaneous changes were also significantly higher in obese children than those with normal BMI. (23). Besides, acanthosis nigricans, a hyperpigmented, papillomatous, velvety cutaneous thickening is also a known dermatological co-morbidity of obesity in children and an independent risk factor for metabolic disorders (hyperinsulinemia, insulin resistance, elevated triglycerides) in obese children (24). Some cutaneous features and skin diseases are associated with genetically induced childhood obesity. Generalised hypopigmentation and acanthosis nigricans are seen in 
Prader-Willi syndrome, regional hyperpigmentation in fragile $\mathrm{X}$ syndrome, atomic eczema in MOMES syndrome, hypohidrosis, nipple hypoplasia in ulnar-mammary syndrome and obesity syndromes which are commonly associated with disorders of pigmentation (25).

Psoriasis, a common chronic immune-mediated inflammatory skin disorder which reduces quality of life of children, is a notable co-morbidity of obesity in children as demonstrated by many studies $(26,27)$

\section{Respiratory system}

New-onset asthma in children has been reported to be associated with increased BMI and females are at a greater risk than their male counterparts (28). In 2019, Deng et al. meta-analysed 18 papers, which studied 73,252 children to assess the association(s) between overweight and obesity on childhood asthma, and the authors discovered that obese children are 1.40 times more likely to develop asthma than non-obese children (29). Obese children are at higher risk of obstructive sleep apnea (OSA), obesity hypoventilation syndrome (OSH) and more severe asthma than children with normal BMI and therefore present with more serious symptoms, require more medication and oxygen when managed in intensive care unit (30).

In the past few decades, the prevalence of obstructive sleep apnea syndrome (OSAS) continues to increase with childhood obesity and therefore is considered to be a co-morbidity. Some of the possible mechanisms are blockage of airway by tonsillar and adenoidal hypertrophy, reduced lung compliance by increased chest wall weight and ventilator instability (31). Not all studies have found a correlation between childhood obesity and obstructive sleep apnea. For example, according to a study on 340 children between 2 and 10 years comprising of 170 children with sleep-disordered breathing and 170 healthy children, done to determine the rate of obesity in these two groups of children, $25.9 \%$ of the study group were obese while $74.1 \%$ were not and it was concluded that there was no significant difference in the two groups (32). The study result might have been skewed by limitations and small study size and therefore may not be enough to reach a general conclusion. Other studies suggested that childhood obesity is linked to OSAS/OSA although the pathophysiology has not been elucidated and obese children with OSAS present more severely and require expert clinical management (28,31). Increased chest and abdominal wall 
weight in obese children result in decreased lung volume ad expiratory flow when subjected to submaximal exercise which further increase inactivity and the risk of other complications as a result of low oxygen saturation (28).

Results from a cohort study of 3960 8-year-old children to determine the relationship between BMI and general health, health related problems, and respiratory infections showed a significant association between obesity and bronchitis (95\% confidence interval) (33). Although non obese children also had respiratory infections during the study but the duration and severity of these infections were more in the obese children (33). This suggests that obesity reduces the body's immune response to infections.

\section{Gastrointestinal system}

Cholelithiasis in children is usually due to haemolytic disorders (34). However, obesity is responsible for $8-33 \%$ of cholelithiasis and this is due to the excretion of cholesterol in bile in excess of the rate of bile acid and phospholipid secretion (34,35). Risk factors for the development of cholelithiasis in obese children are insulin resistance and metabolic syndrome $(34,35)$.

Non-alcoholic steatohepatitis is seen in about $38 \%$ of children with obesity (35). Increased lipolysis and insulin resistance are implicated in the pathogenesis of steatohepatitis in obese children $(34,35)$. Other risk factors for steatohepatitis include diabetes mellitus, hyperlipidaemia, and rapid weight loss (35). Non-alcoholic steatohepatitis can progress to hepatic fibrosis and cirrhosis in severe cases, as previously mentioned above (34-36).

Furthermore, gastro-oesophageal reflux disease (GERD) is more likely to occur in obese children when compared to their normal-weight counterparts (37) and this may be due to the compression of the stomach by the adjacent adipose tissue as seen in obese children with the resulting increase in stomach pressure, opening of the lower oesophageal sphincter and acid reflux into the oesophagus $(37,38)$.

Hypovitaminosis D is also a co-morbidity of childhood obesity and this may be due to the fact that vitamin D is stored excessively in the adipose tissue of obese children (39). More so, children who are overweight or obese are twice more likely to suffer from iron deficiency than those who are 
not $(39,40)$ because obesity inhibit iron absorption by a pathway mediated by inflammation $(39,41)$.

\section{Renal system}

Obesity-related glomerulopathy, end-stage renal disease (ESRD) and chronic kidney disease (CKD) can occur secondary to obesity (42). Obesity induces renal injury by causing hyperinsulinaemia, which via different pathways such as the stimulation of inflammatory cytokines, induction of apoptosis, glomerular hyperperfusion and hyperfiltration, causes renal damage (42). Obesity is not only a risk factor for the development of ESRD and CKD in childhood but also causes a worsening of these conditions $(42,43)$. Other risk factors for the development of ESRD and CKD in obese children are hyperinsulinaemia, hypertension, microalbuminuria, impaired glucose metabolism, and dyslipidaemia (42).

Alport syndromes and Bardet-Biedl syndrome are genetic conditions that have both renal disease and obesity as clinical features (42).

\section{Endocrine system}

Obesity has multiple negative impacts on the glucose-insulin axis and non-esterified fatty acid metabolism. This leads to peripheral insulin resistance and abnormal lipolysis (43). Furthermore, obesity leads to hyperinsulinaemia (43).

Metabolic syndrome is also another endocrine-related co-morbidity of obesity. There is evidence that truncal fat and insulin resistance are the primary causes of this syndrome, where hyperglycaemia, dyslipidaemia, and hypertension exist alongside obesity (44). The components of this syndrome is said to be aggravated with increasing obesity duration. In addition, the concentration of adipokines such as leptin, adiponectin, retinol binding protein-4, chemerin, tumor necrosis factor alpha (TNF $\alpha$ ), and interleukin 6 (IL-6) can be used to predict the risk of an obese child to metabolic syndrome (44). 
Recently, scientists have demonstrated that insulin resistance and increased visceral and total body fat can lead to the development of Impaired Glucose Tolerance, which can progress to type 2 Diabetes Mellitus (45).

Abdominal obesity in children is also associated with non-alcoholic fatty liver disease (NAFLD), which is an early manifestation of ectopic lipid deposition in the liver (46). NAFLD is the most common liver disease among obese children in North America and some other regions (46). The spectrum of NAFLD ranges from steatosis to steatohepatitis to fibrosis and even cirrhosis (46). Other endocrine co-morbidities associated with obesity include Cushing syndrome and hypothyroidism (43).

\section{Reproductive System}

Although, it begins typically in adolescence, polycystic ovarian disease is a common abnormality associated with childhood obesity (47). It is often accompanied by hyperinsulinaemia and hyperandrogenism. Scientists postulate that high circulating insulin levels play a key role in ovarian cyst development due to the anabolic effect of insulin on the ovaries' insulin-like growth factor receptors (47). Moreover, obesity can worsen the symptoms of polycystic ovarian disease by increasing insulin resistance and aggravating metabolic syndrome and diabetes, thus starting a vicious cycle which can lead to infertility (47).

In addition, obesity is a physiological trigger of menarche before 10 years of age (48). Obesity is also associated with oligomenorrhea, amenorrhea and increased risk of complicated pregnancies (48). Furthermore, recent evidences have emerged in support of the theory that childhood obesity is associated with increased risk for impaired oocyte maturation, ovulation, implantation and oocyte quality when puberty is attained (48).

\section{Musculoskeletal system}

Obesity is associated with high frequency and severity of orthopaedic disorders in children. These disorders occur due to increased stress and strain on bones and cartilages which are not designed to accommodate excess weight (49). The most common orthopaedic disorders include bowing of the femurs and tibia which can result in Blount syndrome (overgrowth of the medial aspect of the 
proximal tibial metaphysis) and slipped capital femoral epiphysis due to increased weight on the growth plate of the hip joint (49).

Obesity during the growth spurt phase may increase the risk of fractures from falls (50). This is due to the weight/bone mass imbalance, which also places increased levels of stress on developing bones and joints (50). This can, in turn, result in joint damage and even osteoarthritis in the later years. The recurrence of these fractures and bone disorders can lead to the increased need for complex surgeries and joint replacements in order to manage pediatric trauma. This need, thus, amplifies the physical and financial burden of childhood obesity.

Chronic nonspecific musculoskeletal pain in children has been reported as a common co-morbidity associated with obesity. Children suffering from this kind of pain usually have increased tendencies to be anxious, depressed and take part in lesser activities (51). The sites of pain in this population include the neck and musculoskeletal sites such as the lower back, knees, ankles and feet (51). Additionally, some studies did not specify the site of the reported nonspecific musculoskeletal pain (51).

Recent evidences identified the lower extremities as the most frequent site of this type of pain. An estimated prevalence of this form of lower limb pain among children aged 6-10 years is $24 \%$ (51). Childhood obesity is also associated with foot pain, and with the risk of joint pain increasing by $10 \%$ for every $10 \mathrm{~kg}$ increase in weight and every $3 \%$ increase in BMI (51).

Childhood obesity also correlates with decrease in balance and range of motion (52). This commonly results from excessive anatomical and mechanical strain on the lower limbs, leading to pes valgus, pes planus, genu valgum, etc (52). The dynamic impairment associated with childhood obesity can also manifest as shortening of the hip flexor and knee extensors, especially the quadriceps and psoas muscles (53). This can, in turn, result in hyperlordosis (pronounced curve in the lumbar spine) or retropatellar symptoms (anterior knee pain). 
Childhood obesity is sometimes associated with early onset of arthrosis, especially of the knee, vertebrae and feet (53). The symptoms of arthrosis are accelerated by both the mechanical factors and hormonal changes associated with obesity (53).

Some other well-established orthopaedic comorbidities associated with childhood obesity include Perthes' disease, Osgood Schlatter's disease, Scheuermann's disease and calcaneal apophysitis (54). Again, in these conditions, the lower extremities are mainly affected (54). Moreover, in obese children with diabetes mellitus, Charcot foot and degenerative disorders of the musculoskeletal system is sometimes present (55).

\section{Conclusion}

With more children becoming obese every year, obesity had reached epidemic proportions and is a major public health challenge. As demonstrated by this review, obesity is a multi-systemic disease with affectation of all body systems. Some of these co-morbidities occur in childhood while others are delayed till later life. Nevertheless, obesity remains a leading cause of preventable deaths.

Obesity is a preventable disease. As such, at the individual level, children should be encouraged to eat healthier foods and do regular exercise. At the community level, policies that support obesity prevention should be implemented (1).

Co-morbidities associated with obesity should be routinely investigated and promptly treated.

\section{Acknowledgement}

The authors received no funding for this research.

\section{Conflict of interest}

The authors declare no conflict of interest.

\section{Authors contribution}

All authors contributed equally to this research 


\section{Reference}

1. World Health Organization. Obesity and Overweight (Internet). 2020 (cited 2020 Sep 19). Available from: https://www.who.int/news-room/fact-sheets/detail/obesity-and-overweight

2. Mccrindle BW. Cardiovascular Consequences of Childhood Obesity. Can J Cardiol. 2015;31(2):124-30.

3. Ippisch HM, Daniels SR. Hypertension in overweight and obese children. 2008;25:177-82.

4. Litwin SE. Childhood Obesity and Adulthood Cardiovascular Disease\&lowast; J Am Coll Cardiol. 2014;64(15):1588-90.

5. Sorof JM, Lai D, Turner J, Poffenbarger T, Portman RJ. Overweight, Ethnicity, and the Prevalence of Hypertension in School-Aged Children. 2020;113(3).

6. Raj M. Review Article Obesity and cardiovascular risk in children and adolescents. 2020;16(1):13-20.

7. Liao D, Rodrı SM, He F, Bixler EO. Childhood Obesity and Autonomic Dysfunction : Risk for Cardiac Morbidity and Mortality. 2014;

8. Taquari R. Childhood obesity and its cardiovascular implications: a current view. 2014;31(1):1-5.

9. Raghuveer G. Lifetime cardiovascular risk of childhood obesity 1-4. 2010;91:1514-9.

10. Weihrauch-blüher S, Wiegand S. Risk Factors and Implications of Childhood Obesity. 2018;254-9.

11. Perlaki G, Molnar D, Id PAMS, Ahrens W, Wolters M, Eiben G, et al. Volumetric gray matter measures of amygdala and accumbens in childhood overweight / obesity. 2018;117.

12. Health A. Psychological consequences of childhood obesity : psychiatric comorbidity and prevention. 2016;125-46.

13. Hussein R, Mohammed RA, Ahmed IH. Psychological impact of obesity in children. $2020 ; 17-21$. 
14. Strauss RS. Childhood obesity and self-esteem. Pediatrics. 2000;105(1).

15. Ebbeling C, Pawlak D, Ludwig D. Childhood Obesity: Public-Health Crisis, Common Sense Cure. Lancet. 2002;360(9331):473.

16. Reiff MI. Are overweight children unhappy? J Dev Behav Pediatr. 2001;22(1):82.

17. Daniels SR. Complications of obesity in children and adolescents. Int J Obes. 2009;33:S605.

18. Agranat-meged AN, Deitcher C, Goldzweig G, Stein M. Childhood Obesity and Attention Deficit / Hyperactivity Disorder : A Newly Described Comorbidity in Obese Hospitalized Children. 2005;1999-2002.

19. Kalarchian MA, Marcus MD, Kalarchian MA, Marcus MD. Psychiatric comorbidity of childhood obesity Psychiatric comorbidity of childhood obesity. 2012;0261.

20. Halfon N, Larson K, Slusser W. Associations Between Obesity and Comorbid Mental Health, Developmental, and Physical Health Conditions in a Nationally Representative Sample of US Children Aged 10 to 17. Acad Pediatr. 2013;13(1):6-13.

21. Nino M, Ph D, Franzese A, Perrino NR, Ph D, Balato N. The Effect of Obesity on Skin Disease and Epidermal Permeability Barrier Status in Children. 2012;29(5):567-70.

22. Paller A, Jaworski JC, Simpson EL, Boguniewicz M, Russell JJ, Block JK, et al. Major Comorbidities of Atopic Dermatitis: Beyond Allergic Disorders. Am J Clin Dermatol. 2018;19(6):821-38.

23. Mirmirani P, Carpenter DM. Skin Disorders Associated with Obesity in Children and Adolescents : A Population-Based Study. 2014;31(2):183-90.

24. Guran T, Turan S, Akcay T, Bereket A. PDFlib PLOP : PDF Linearization, Optimization, Protection Page inserted by evaluation version Significance of acanthosis nigricans in childhood obesity.

25. Millington GWM. Obesity, genetics and the skin. 2012;(February):50-6.

26. Bronckers IMGJ, Paller AS, Geel MJ, Kerkhof PCM, Seyger MMB. Psoriasis in Children and Adolescents: Diagnosis, Management and Comorbidities. Pediatr Drugs. 
2015;17(5):373-84.

27. Koebnick C, Black MH, Smith N, Der-sarkissian JK, Porter AH, Jacobsen SJ, et al. The Association of Psoriasis and Elevated Blood Lipids in Overweight and Obese Children. J Pediatr. 2011;159(4):577-83.

28. Durbin C, Egan R, Gervasi K, Nadeau N, Neal E, Reich S, et al. in children. 2017;30(9):303.

29. Deng X. Association between overweight or obesity and the risk for childhood asthma and wheeze : An updated meta - analysis on 18 articles and 73252 children. 2019;(March).

30. Fiorino EK, Brooks LJ. Obesity and Respirator y Diseases in Childhood. Clin Chest Med. 2009;30(3):601-8.

31. Arens R, Muzumdar H. Childhood obesity and obstructive sleep apnea syndrome. 2010;(104):436-44.

32. Esteller-moré E, Castells-vilella L, Segarra-isern F, Argemí-renom J, Sue U. Childhood Obesity and Sleep-related Breathing Disorders \&. Acta Otorrinolaringol (English Ed. 2012;63(3):180-6.

33. Wijga AH, Scholtens S, Bemelmans WJE, Jongste JC De, Kerkhof M, Schipper M, et al. Comorbidities of obesity in school children : a cross-sectional study in the PIAMA birth cohort. 2010;

34. Must A, Strauss RS. Risks and consequences of childhood and adolescent obesity. Int J Obes. 1999;23:S2-11.

35. Banerjee A, Schuster D. Comorbidities of Childhood Obesity. In: Childhood Obesity. 2012.

36. Tominaga K, Kurata JH, Chen YK, Fujimoto E, Miyagawa S, Abe I, et al. Prevalence of fatty liver in Japanese children and relationship to obesity. Dig Dis Sci. 1995;40(9):20029.

37. Malaty H. Obesity and gastroesophageal reflux disease and gastroesophageal reflux symptoms in children. Clin Exp Gastroenterol. 2009;31. 
38. Fass R. The pathophysiological mechanisms of GERD in the obese patient. Dig Dis Sci. 2008;53(9):2300-6.

39. Han JC, Lawlor DA, Kimm SYS. Childhood Obesity - 2010 : Progress and Challenges. Lancet. 2010;375(9727):1737-48.

40. Nead KG, Halterman JS, Kaczorowski JM, Auinger P, Weitzman M. Overweight children and adolescents: A risk group for iron deficiency. Pediatrics. 2004;114(1):104-8.

41. McClung JP, Karl JP. Iron deficiency and obesity: The contribution of inflammation and diminished iron absorption. Vol. 67, Nutrition Reviews. 2009;100-4.

42. Savino A, Pelliccia P, Chiarelli F, Mohn A. Obesity-related renal injury in childhood. Vol. 73, Hormone Research in Paediatrics. 2010;303-11.

43. De Jong PE, Verhave JC, Pinto-Sietsma SJ, Hillege HL. Obesity and target organ damage: The kidney. Int J Obes. 2002;26:S21-4.

44. Papaetis GS, Papakyriakou P, Panagiotou TN. Central obesity, type 2 diabetes and insulin: exploring a pathway full of thorns. Archives of Medical Science : AMS. 2015 Jun;11(3):463-482.

45. Weiss R, Bremer AA, Lustig RH. What is metabolic syndrome, and why are children getting it?. Ann N Y Acad Sci. 2013;1281(1):123-140.

46. Al-Goblan AS, Al-Alfi MA, Khan MZ. Mechanism linking diabetes mellitus and obesity. Diabetes Metab Syndr Obes. 2014;7:587-591. Published 2014 Dec 4.

47. EJ, Kim HJ. Nonalcoholic fatty liver disease in obese and nonobese pediatric patients. Korean J Pediatr. 2019;62(1):30-35.

48. Anderson AD, Solorzano CM, McCartney CR. Childhood obesity and its impact on the development of adolescent PCOS. Semin Reprod Med. 2014;32(3):202-213.

49. Freedman DS, Khan LK, Serdula MK, et al. The relation of menarcheal age to obesity in childhood and adulthood: the Bogalusa heart study. BMC Pediatr. 2003;3:3.

50. Wills M. Orthopedic complications of childhood obesity. Pediatr Phys Ther. 2004;16(4):230235. 
51. Taylor ED, Theim KR, Mirch MC, et al. Orthopedic complications of overweight in children and adolescents. Pediatrics. 2006;117(6):2167-2174.

52. Smith SM, Sumar B, Dixon KA. Musculoskeletal pain in overweight and obese children. Int J Obes (Lond). 2014;38(1):11-15.

53. Merder-Coşkun D, Uzuner A, Keniş-Coşkun Ö, Çelenlioğlu AE, Akman M, Karadağ-Sayg1 E. Relationship between obesity and musculoskeletal system findings among children and adolescents. Turk J Phys Med Rehabil. 2017;63(3):207-214.

54. Pandey R, Kumar N, Paroha S, et al. Impact of obesity and diabetes on arthritis: An update. Health (Irvine Calif). 2013;5(1):143-156.

55. Chan G, Chen CT. Musculoskeletal effects of obesity. Curr Opin Pediatr. 2009;21(1):65-70.

56. Obalum DC, Fiberesima F, Eyesan SU, Ogo CN, Nzew C, Mijinyawa M. A review of obesity and orthopaedic surgery: the critical issues. Niger Postgrad Med J. 2012;19(3):175-180. 